Tanja Heiden*

\title{
Der Translator als kreativer bilingualer und bikultureller Mittler oder Wie er sein Handeln zwischen konzeptueller Past-fiction und Science- fiction $^{1}$ rechtfertigen kann
}

\section{Relevanz des Scenes-and-Frames Ansatzes für den Übersetzungsunterricht ${ }^{2}$}

\section{Überblick}

Der Artikel ist primär ein Beitrag zur Operationalisierung des scenes-and-frames Konzepts in der Translationswissenschaft. Nach einer Einführung der zentralen Begriffe werden diese auf einen konkreten Textkorpus bezogen und ihre Relevanz für die Beschreibung des Übersetzungsprozesses dadurch belegt. Daraufhin wird eine Frametypologie vorgeschlagen, die im Verlauf der Untersuchung auch empirisch getestet wurde. Und schliesslich wird auf der Grundlage des vorgelegten Ansatztes noch eine mögliche kognitive übersetzungsprozedurale Konzeption vorgestellt.

\section{Einführung}

Denn wir fingen an zu begreifen, dass Lesen nicht ein Ausflug ins Grüne ist, bei dem man fast zufällig rechts oder links eine poetische Blume pflückt, hier einen Hahnenfuss und dort einen Weissdorn, versteckt im Dünger der strukturellen Füll- und Flickwörter, sondern

1 Hier als Rekonstruktion der Vergangenheit bzw. Vorwegnahme der Zukunft zu verstehen.

2 Überarbeitete und erweiterte Fassung meines Beitrags an der vom 13. - 15. März 2003 an der Business School of Aarhus (Dänemark) abgehaltenen Eurokonferenz zum Thema „Knowledge Systems in Text and Translation“.

* Tanja Heiden

ETI

Université de Genève

40, Boulevard du Pont-d'Arve

CH-1211 Genève 4

Hermes, Journal of Linguistics no 33-2004 
dass man den Text als etwas Ganzes nehmen muss, das auf verschiedenen Stufen mit Leben erfüllt ist. (U. Eco in Die Bücher und das Paradies. Über Literatur)

Im vorliegenden Beitrag soll ein translationsrelevantes ganzheitliches Konzept der Textanalyse und -(re)produktion vorgestellt werden, das im Übersetzungssunterricht (Vannerem/Snell-Hornby 1986) seit den frühen neunziger Jahren erfolgreich zum Einsatz kommt: Die Rede ist vom Scenes-and-frames Modell.

Diese ursprünglich in der Psycholinguistik bei Charles Fillmore (1977) anzusiedelnde Theorie der Bedeutung - eine Weiterentwicklung der Prototypen-Semantik (Rosch 1973) - die später in die Translationswissenschaft übernommen und translationsrelevant aufgearbeitet wurde (Vannerem/Snell-Hornby 1986; Snell-Hornby 1988; Vermeer/Witte 1990), soll im nachstehenden Kapitel 2 zunächst kurz erläutert, auf die Translationswissenschaft bzw. den -prozess angewandt und mit Beispielen aus unserem Textkorpus besser veranschaulicht werden.

In Kapitel 3 geht es dann darum, eine Konzeptevaluierung vorzunehmen und den Stellenwert des Scenes-and-frames Ansatzes in der Translationswissenschaft bzw. im -unterricht aufzuzeigen. Ausgehend von einer durchaus ernstzunehmenden Kritik (Gerzymisch-Arbogast 1994: 77), derzufolge die Schwäche des Scenes-and-frames Ansatzes darin liege, nicht zu erklären, wie frames - die sprachlichen Kodierungen vs. den scenes, den Szenen hinter den frames - denn eigentlich erstellt und voneinander abgegrenzt werden können, möchte ich anschliessend in Kapitel 4 eine Frame-Typologie vorschlagen, die uns bei einer systematische(re)n Identifikation und Abgrenzung der in einem Text vorkommenden frames im Übersetzungsunterricht behilflich sein kann.

Und schliesslich soll auf der Grundlage des dargelegten Ansatzes noch eine mögliche kognitive übersetzungsprozedurale Konzeption vorgestellt werden. In diesem Sinne möchte ich ein Verfahren vorschlagen, mit dem der Transfer von scenes und frames aus der Ausgangskultur in die Zielkultur nicht mehr ausschliesslich nur auf Textebene, sondern ganzheitlich auf (konzeptueller) Systemebene unter Berücksichtigung des Hintergrundwissens sowie der Erwartungen des Zielpublikums operationalisierbar wird. 


\section{Untersuchungsgegenstand sowie zum Scenes-and- Frames Ansatz in der Translationswissenschaft}

\subsection{Methodologie}

Bevor ich auf die methodologischen Ansätze zu sprechen komme, die bei der vorliegenden Betrachtung dienlich waren, sollen zunächst einmal die zur Analyse herangezogenen Texte näher beschrieben werden.

\subsubsection{Beschreibung des Textkorpus}

Angesichts der Tatsache, dass sich diese Untersuchung mit der Übertragung von scenes und frames beschäftigt und diese beiden Konzepte - worauf wir später auch noch näher eingehen werden - stets auch kulturabhängig sind, habe ich bei der Zusammenstellung des Textkorpus darauf geachtet Texte miteinzubeziehen, in denen sprachliche Elemente und kultureller bzw. situativer Kontext eng miteinander verflochten sind. Dies ist besonders bei literarischen Texten der Fall. Weiters habe ich bei meiner Auswahl humoristischen Texten, genauer gesagt, ironisch-satirischen Texten den Vorzug gegeben, da Humor bekannterweise besonders kulturgebunden ist und humoristische Stellen bei der Übertragung von scenes und frames, die kulturell unterschiedlich ablaufen können (vgl. Feyrer, 2001: 42), manchmal Übersetzungsschwierigkeiten bereiten. Schliesslich sollten die Texte in einer ersten Annäherung das Sprachenpaar italienisch/deutsch betreffen, da diese Sprachenkombination im Bereich der Translationswissenschafte allgemein und im Bereich der Studien zu Humor und Translation insbesondere m. A. n. bisher - von einigen wenigen Ausnahmen einmal abgesehen (vgl. Albrecht 1998) - eher stiefmütterlich behandelt wurde.

Unter Berücksichtigung all dieser Faktoren sowie einer persönlichen Vorliebe habe ich mich somit für einen der bekanntesten Schriftsteller unserer Zeit entschieden: Umberto Eco - in dessen Rolle als Polemiker, kritischer Beobachter seiner und unserer Gegenwart und Verfasser zahlreicher italienischer Satiren und Glossen, abgefasst in Il secondo diario minimo (1992 bei Bompiani erschienen) und deren deutsche Übersetzungen in Wie man mit einem Lachs verreist und andere nützliche Ratschläge (Burkhard Kroeber und Günter Memmert, 1993 im Carl Hanser Verlag veröffentlicht) sowie in Gesammelte Streichholzbriefe (Burkhard Kroeber, 1990/95 ebenfalls im Carl Hanser Verlag 
herausgegeben). Die im italienischen Werk vereinigten Texte sind in den achtziger Jahren zu verschiedenen Anlässen für das römische Nachrichtenmagazin $L$ 'Espresso geschrieben worden, grösstenteils für die dort 1986 begonnene Rubrik La Bustina di Minerva (von denen einige dann auch als Streichholzbriefchen in DIE ZEIT erschienen sind). „Kritisch erhellt Eco darin Ärgernisse des Alltags, enervierende Zeitgeist-Phänomene und liebenswerte Untugenden seiner Mitmenschen" ist im Klappentext zu den Gesammelten Streichholzbriefchen zu lesen. Umberto Eco beschreibt in diesen Essays mit viel Witz und Scharfsinn die kleinen Tücken des Alltags, die jeder kennt, wie etwa die Benutzung von kleckernden Kaffeekannen oder den ärgerstiftenden Umgang mit Gebrauchsanweisungen, er analysiert aber auch wichtige politische, soziologische und philosophische Fragen unserer Epoche.

\subsubsection{Beschreibung der methodologischen Ansätze}

Folgende übersetzungstheoretischen Ansätze bildeten für diese Untersuchung eine Grundlage:

\section{Skopostheorie}

Die Skopostheorie nach Reiss und Vermeer (1978) stellt als „komplexe Handlungstheorie“3 bei einer Betrachtung von sprachlichen, kulturellen sowie situativen Elementen im kontrastiven Vergleich eine u. A. n. wahrscheinlich generell oft geeignete übersetzungswissenschaftliche methodologische Grundlage dar. So nimmt der Translator - worauf wir auch später noch einmal eingehender zu sprechen kommen - als kreativer Empfänger die vom Autor der Ausgangssprache in einer bestimmten Situation und zu einem bestimmten Zweck geschaffenen Bedeutungsinhalte und mentalen Konzepte auf, arbeitet diese übersetzungsrelevant um und übermittelt diese anschliessend angemessen in die Zielsprache. Der Skopos oder das „Translationsziel“ (Vermeer und Witte, 1990: 46) bzw. die Funktion des Textes ist meine ich eine Grundlage, auf welcher der Translator beim Transfer der beschriebenen Elemente arbeiten kann.

3 Der Begriff „Komplexe Handlungstheorie“ wird hier in Anlehnung an Feyrer (2001: 10) in der Definition von Reiss und Vermeer (1991: 95) verstanden. 


\section{Scenes-and-frames Theory}

Als zweiter Ansatz soll die auf Fillmore (1977) basierende scenes-andframes theory herangezogen werden, die gleich in den folgenden Abschnitten eingehender beschrieben werden soll.

\subsection{Terminologie des Scenes-and-frames Konzepts - Kurze Einführung der zentralen Begriffe}

\subsubsection{Von der Wahrnehmung (frame) zur Vorstellung (scene)}

Wie vermutlich allgemein bekannt, aber auch bei Schwarze (1992: 75 ff.) nachzulesen, sind die zentralen Fragen der Kognitionswissenschaften Fragen nach dem Wissen von Menschen. Eine der Grundannahmen der Kognitionswissenschaften ist, dass wir uns die uns umgebende und wahrnehmbare Welt auf eine spezifische Art mental vorstellen und dass bestimmte Verarbeitungsprozesse auf diesen mentalen Strukturen ablaufen, die auch komplexes Handeln, wie z. B. Übersetzen, ermöglichen. Aber woraus setzen sich diese mentalen Strukturen nun zusammen? Als Einheiten unserer strukturellen Kognition werden von den meisten Kognitionsforschern Konzepte und Schemata betrachtet, erstere als elementare, mentale Organisationseinheiten und letztere als komplexe, mentale Organisationseinheiten, zumal Konzepte im Langzeitgedächtnis bekanntlich nicht isoliert abgespeichert, sondern durch verschiedene Relationen miteinander verknüpft sind.

\subsubsection{Von scenes...}

Komplexe Wissensstrukturen, welche u. a. die Erfahrung repräsentieren, die ein Mensch im Laufe seines Lebens macht, werden bei Fillmore metaphorisch als scenes bezeichnet. So sieht Fillmore (1977: 63) scene nicht nur im herkömmlichen Sinn visuell sondern weiter gefasst als $\mathrm{fa-}$ miliar kinds of interpersonal transactions, standard scenarios [...] enactive experiences [...] any kind of coherent segment [...] of human beliefs, actions, experiences or imaginings. Bei Vannerem/Snell-Hornby (1994: 185) werden scenes auf den Übersetzungsprozess bezogen als erlebte Situationen beschrieben und bei Vermeer und Witte (1990: 51) stellen sie kulturspezifische sich im Kopf eines Menschen aufbau- 
ende und dann existierende mehr oder minder schemenhafte oder detaillierte und damit mehr oder minder komplexe Vorstellung aufgrund von Wahrnehmungen dar. Scenes sind in diesem Beitrag als Szenen hinter einer sprachlichen Kodierung zu begreifen und bei der Verwendung des Begriffs „Szene“ denkt man sofort an mentale Bilder und Vorstellungen.

\section{... und frames}

Auch hier verwendet Fillmore (1977: 63) den Begriff frame viel weiter gefasst als vielfach angenommen, nämlich: for referring to any system of linguistic choice - the easiest being collections of words, but also including choices of grammatical rules or grammatical categories - that can get associated with prototypical instances of scenes. Unter frame verstehen wir hier, translationsrelevant beschrieben, jede sprachliche Kodierung, die „Versprachlichung“ von Denkvorstellungen oder, mit anderen Worten, die sprachlich erfasste bzw. verbalisierte scene.

\subsubsection{Anwendung des Scenes-and-frames Ansatzes auf den Übersetzungsprozess}

Wenn Vannerem/Snell-Hornby (1994: 185) schreiben, dass nach Fillmore der Kommunikations- und Verstehensprozess so ablaufe, dass wir zu jeder sprachlichen Form (frame) zunächst mittels eigener Erfahrung bzw. einer Situation (scene) Zugang finden, die für uns persönlich von Bedeutung ist, dann lassen sich die während des Übersetzungsprozesses ablaufenden mentalen Vorgänge beim Translator wie folgt beschreiben: Dieser interpretiert zunächst die durch den Ausgangstext aktivierten scenes, bringt sie mit seiner persönlichen Erfahrung in Verbindung und versucht, die in der Zielsprache und -kultur geeigneten (adäquat im Sinne des intendierten Zwecks) scenes zunächst für sich selbst aufzurufen, um sie anschliessend für den Zieltextrezipienten aufzurufen und sie schliesslich diesem zugänglich zu machen.

Ein sehr gutes Beispiel für diesen Vorgang und speziell für das Einbringen von persönlicher Erfahrung bzw. Hintergrundwissen durch Eco's Übersetzer finden wir in folgendem Essay, den Eco mit Come fare le vacanze intelligenti betitelt, von Burkhard Kroeber übersetzt mit: „Wie man intelligente Ferien macht“. In dieser Glosse macht sich Eco über so manche politische und kulturelle Wochenzeitschrift lustig, 
die beim Herannahen der Sommerferien ihren Lesern eine Liste intelligenter Bücher empfehlen, mit denen sie auf intelligente Weise intelligente Ferien verbringen sollten.

Per chi avrà lunghe ore in spiaggia consiglierei la Ars magna lucis et umbrae di padre Athanasius Kircher, affascinante per chi sotto i raggi infrarossi voglia riflettere sui prodigi della luce et degli specchi. L'edizione romana del 1645 è ancora reperibile in antiquariato che cifre indubbiamente inferiori che quelle che Calvi ha esportato in Svizzera.

Eco (1992: 75)
Wer lange Stunden am Strand zu verbringen gedenkt, sollte sich die Ars magna lucis et umbrae von Pater Athanasius Kircher vornehmen, eine faszinierende Lektüre für den, der unter den Ultraviolettstrahlen über die Wunder des Lichts und der Spiegel nachdenken will. Die römische Ausgabe von 1645 ist noch in Antiquariaten erhältlich für Summen weit unter denen, die seinerseits der Banquier Calvi in die Schweiz ausgeführt hat.

Kroeber (1993: 30/31)

Wie dem aufmerksamen Leser sicherlich aufgefallen ist, hat der Eigenname Calviin diesem Kontext beim Übersetzer durch das Zusammenspiel von Bottom-Up- und Top-down-Prozessen bestimmte Assoziationen ausgelöst. Er hat hier auf sein Weltwissen zurückgegriffen und vor den Eigennamen Calvi die Berufsbezeichnung „Banquier“ hinzugesetzt, was zudem überaus treffend war, weil dadurch auch der so wesentliche ironische Effekt des Ausgangstextes noch mehr verstärkt wurde.

\subsubsection{Zu den wesentlichen Merkmalen von scenes und frames}

Scenes und frames - und das ist ein wichtiges Merkmal beider Konzepte - laufen kulturell unterschiedlich ab (vgl. Feyrer, 2001: 42). Übersetzungsprobleme ergeben sich in vielen Fällen jedoch eher auf Grund kulturell divergierender scenes, wenn nämlich ein Rezipient der Zielsprache mit einer sprachlichen Kodierung nicht dieselben kulturabhängigen Vorstellungen verbindet wie derjenige der Ausgangssprache, an den der Text ursprünglich gerichtet war. Diese kulturellen Unterschiede leben vom Impliziten, das der Translator richtig erkennen und umsetzen muss. In der Übersetzung - die Rede ist hier vom Produkt (dem Translat) - müssen dann eben andere scenes entstehen und dafür auch andere frames verwendet werden. 
Für die charakteristische Anpassung eines frames (und der dahinter stehenden scene) an die Kulturspezifik lassen sich, wie sich der Leser bestimmt denken kann, zahlreiche Textbeispiele finden.

Im selben Essay etwas weiter heisst es etwa:

Un giovane invece tenti viaggi a biglietto
forfettario per l'Europa, in seconda classe,
e che debba quindi leggere in quei treni
dai corridoi completamente affollati, dove
si sta in piedi con un braccio fuori dal
finestrino, potrebbe condur seco almeno
tre dei sei volumi einaudiani del Ra-
musio, leggibili tenendone uno tra le
mani, l'altro sotto il braccio, il terzo tra
l'inguine e la coscia.
Eco (1992: 75/76)

\begin{abstract}
Ein junger Mensch hingegen, der sich auf Pauschalpreis-Reisen durch Europa begibt, in der zweiten Klasse, so dass er in Zügen lesen muss, in denen die Korridore total überfüllt sind und man eingezwängt stehen muss, einen Arm aus dem Fenster gehängt, könnte sich mindestens drei Bände der sechsbändigen Einaudi-Ausgabe der „Navigazioni et viaggi» des humanistischen Geographen Gian Battista Ramusio mitnehmen, die sich gut lesen lassen, wenn man einen Band in der Hand hält, den zweiten unter den Arm geklemmt und den dritten zwischen die Schenkel.
\end{abstract}

Kroeber (1993: 31)

An dieser Stelle hielt es der Übersetzer im Hinblick auf das Vorwissen des deutschen Zielpublikums für angebracht, diesem die Zusatzinformation $\mathrm{zu}$ geben, dass es sich bei den im Ausgangstext angesprochenen drei Bänden der sechsbändigen Einaudi-Ausgabe von Ramusio um dessen Werk der „Navigazioni et viaggi“ handelt (glz. Anspielung auf die derzeit bei jungen Leuten so beliebten Interrail-Reisen) und dass Ramusio ein humanistischer Geograph war. Der deutschsprachige Leser, der diese Information in seinem Hintergrundwissen nicht abrufbar gespeichert gehabt und der von Kroeber diesen Zusatzframe nicht erhalten hätte, hätte einen Teil der intertextuellen Ironie hier nicht verstanden, da er den Zusammenhang zwischen quer durch Europa trampenden Jugendlichen und dem Titel des Buches sowie dem Beruf des Autors nicht hätte herstellen können.

Eine weitere Passage, an der Burkhard Kroeber bemüht war beim deutschen Zielpublikum, durch eine sprachliche Formulierung - einen frame - die in etwa selbe Denkvorstellung - eine scene - auszulösen wie dies beim Zielpublikum der Ausgangssprache und -kultur vermutlich der Fall war und er deshalb - die Kulturspezifik des Ziellandes respektierend treffend adaptiert hat und er einen AS-frame durch einen ganz 
anderen ZS-frame (völlige Nicht-Übereinstimmung) ersetzt hat, ist die folgende, immer noch im gleichen Textabschnitt zu findende:

Ci pare pertanto offensivo o quanto meno paternalistico offendere il lettore consigliandogli, che so, l'originale tedesco delle Affinità elettive, il Proust della Pléiade o le opere latine del Petrarca.

Eco (1992 : 75)
Es mutet uns in der Tat beleidigend oder zumindest sehr paternalistisch an, den Lesern Werke wie, was weiss ich, das englische Original des „Tristram Shandy», den Proust der Pléiade oder die lateinischen Schriften Petrarcas zu empfehlen.

Kroeber (1993: 30)

Freilich wäre es an dieser Stelle widersinnig gewesen, einem deutschen Leser das deutsche Original von Geoethes Wahlverwandtschaften zu empfehlen und zielsicher hat Kroeber - an dieser Stelle die Erwartungen seines Zielpublikums vorwegnehmend - einen entsprechenden frame, hier aus dem englischsprachigen Kulturraum, gewählt, der im Deutschen in etwa die selbe scene evoziert wie die scene hinter dem frame im italienischen Ausgangstext. Eine 1:1 Substitution des AS-frames durch das selbe ZS-frame ohne Berücksichtigung der dabei evozierten scene beim ZS-Leser hätte bei diesem eine verfremdende Wirkung zur Folge gehabt.

Wichtig ist an dieser Stelle darauf hinzuweisen und hiermit schliesse ich mich Vannerem/Snell-Hornby (1994: 190; zit. Hönig 1986; Stolze 1982) an, dass ein scenes-and-frames Ansatz eine Textanalyse auf semantisch-grammatikalischer und syntaktisch-stilistischer Ebene keineswegs überflüssig macht. Im Gegenteil: Der Übersetzer sollte sich auch bei seinen Assoziationen ständig auf die sprachlichen Informationen berufen, die ihm der Text liefert, sich immer darauf rückbeziehen, sich beim Text gewissermassen „rückversichern“.

In Übereinstimmung mit den zitierten Arbeiten ist es ausserdem von Interesse zu betonen - und dies stellt neben der Kulturabhängigkeit von scenes und frames ein weiteres implizites Merkmal der hier besprochenen Konzepte dar - dass scenes und frames einander wechselseitig bedingen. Das heisst, dass ein frame eine scene aktiviert, eine scene wieder eine andere scene mit einem entsprechenden frame und die sprachliche Formulierung (der frame) wiederum eine oder mehrere bestimmte scenes beim Rezipienten aufrufen kann. Insofern bezeichnet 
Feyrer (vgl. 2001: 15) scenes und frames treffend als kohärenzstiftende Faktoren im Text.

Zusammenfassend gilt für scenes und frames - auf den Übersetzungsprozess angewandt - somit Folgendes: Ziel einer jeden Übersetzung sollte sein, eine durch einen frame hervorgerufene scene des Ausgangstextes (AT) so in einen frame des Zieltextes (ZT) zu enkodieren, dass der frame des ZT und die für den Rezipienten damit evozierte scene der vom Autor intendierten scene des AT trotz individueller Betrachtung, Erwartung und Hintergrundwissen immer noch möglichst genau entspricht.

\section{Evaluierung und Relevanz des Scenes-and-frames Ansatzes für die Übersetzungswissenschaft}

Eine scene ruft, wie wir erfahren haben, einen frame auf. Ein frame kann aber ebensogut einen weiteren frame aktivieren. Anders ausgedrückt, die sprachliche Kodierung des AT kann eine neue sprachliche Kodierung in der ZS schon zwingend nahe legen (vgl. Hönig und Kussmaul 1982: 26ff. zit. in Vannerem/Snell-Hornby 1994: 191).

In diesem Fall ist es die Aufgabe eines Translators, sorgfältig zu prüfen, ob diese quasi automatische frame-frame-Assoziation nicht etwa durch eine verwandte Bedeutung oder durch ähnlich geschriebene andere frames ausgelöst wurde. Ausserdem bedeutet die Tatsache, dass sofort ein anderer frame erfasst wurde, noch lange nicht, dass diese erstgefundene Lösung auch die beste, $d$. h. die adäquateste bzw. angemessenste im Sinne des intendierten Zwecks ist ${ }^{4}$. Auch hier gilt es wieder, sich beim Text rückzuversichern. Nun kommt es in der Unterrichtspraxis jedoch mitunter vor (und dies ist v. a. bei Studierenden der Translationswissenschaften in den ersten Semestern, wie ich feststellen konnte, häufig der Fall), dass die durch den Ausgangstext aktivierten und vom Translator in seiner Rolle als erster Modellleser interpretierten scenes nicht unbedingt dieselben sind wie diejenigen, die vom

4 Auf die übersetzungstheoretischen Begriffe der Adäquatheit, Angemessenheit oder Äquivalenz soll an dieser Stelle nicht näher eingegangen werden, da diese eine eigene Abhandlung verdienen würden. 
Autor auch beabsichtigt waren ${ }^{5}$. Diese Fehlinterpretation ist in den meisten Fällen auf noch unzureichende Fremdsprachenkenntnisse zurückzuführen, da der Studierende in diesem Lernstadium nur allzuoft als Nicht-Muttersprachler handelt und in diesem Sinne hinter einem frame nicht dieselben scenes aktiviert, wie es der Muttersprachler tun würde oder wie es der Autor beabsichtigt hat. Auch hier kann man wieder sehen, dass die von einem frame aktivierten scenes sehr eng mit der Soziokultur des jeweiligen Sprachbenutzers verbunden sind (vgl. Vannerem/Snell-Hornby 1994: 190, Hönig \& Kussmaul, 1982).

Um solchen Übersetzungsfehlern entgegenzuwirken, ist - wie in der einschlägigen Literatur zwar schon mehrmals betont wurde aber nie oft genug wiederholt werden kann - eine optimale Kenntnis der Fremdsprache(n) und -kulturen vonnöten. In diesem Sinne ist der Translator in einem ersten Schritt u. A. n. als bilingualer (bzw. multilingualer) und bikultureller (bzw. multikultureller) Mittler zu beschreiben.

Die Anwendung des scenes-and-frames Ansatzes auf den Übersetzungsprozess versetzt den Translator zum einen in die Rolle eines kreativen Empfängers, der die von einem Text gelieferten AS-frames (und die dahinter verborgene AS-scene) aufnimmt, interpretiert und verarbeitet - indem er sein eigenes Hintergrundwissen (prototypisches Weltwissen, vgl. Vannerem/Snell-Hornby 1994: 192) sowie seine eigenen Erwartungen einbringt - und zum anderen in die eines kreativen Senders, der diese Informationen unter Berücksichtigung des Hintergrundwissens sowie der Erwartungen des Rezipienten in Form eines anderen ZS-frame (und die wieder dahinter verborgene ZS-scene) in die ZK überträgt. Der Übersetzer dekodiert und enkodiert frames und scenes und ist unter diesem Blickwinkel nicht nur als bilingualer und bikultureller sondern generell als kreativer Mittler zu bezeichnen.

Wie Feyrer (vgl. 2001: 68) anmerkt und dies auch an zahlreichen Stellen im Textkorpus feststellbar war, gilt für scenes und frames weiters, dass scenes oft konventionalisierte frames aufrufen und diese im Translat dann meistens als idiomatische Wendungen auftauchen.

5 Siehe hierzu auch die von U.Eco beschriebene Triade der Intentionen bestehend aus: Autor-, Werk- und Leserintention in: U. Eco (2003): Die Bücher und das Paradies. Über Literatur. Carl Hanser Verlag: München 
Auch hierzu gleich wieder ein Beispiel, dieses Mal aus Eco's Essay: Come sostituire una patente rubata, im Deutschen übersetzt mit: Wie man einen verlorenen Führerschein ersetzt. Wütend und sarkastisch erzählt uns Eco eine Konfrontation mit den italienischen Behörden, nachdem ihm einmal sein Führerschein abhanden gekommen war und er einen neuen beantragen wollte, wofür er den Behörden die Nummer des alten Führerscheins bekanntgeben musste, diese aber nirgendwo anders notiert war als auf dem alten verlorengegangenen Führerschein selbst.

Penso che il lettore si renda conto di quale pericolo corebbero infatti le istizuzioni se il numero della mia patente venisse communicato a cani e porci. Ghaddafi e il KGB non aspettano altro. Dunque, top secret.

Eco (1992: 79)
Ich denke der Leser wird sich darüber im klaren sein, welche Gefahr in der Tat die Behörden liefen, würden sie meine Führerscheinnummer einfach an Hinz und Kunz weitergeben: Ghaddafi und der KGB warten doch nur darauf. Also top secret.

Kroeber (1993: 37)

Die AS-scene, d. h. die Vorstellung, dass die Führerscheinnummer an xbeliebige Leute (a cani e porci) weitergegeben wird, hat im Deutschen gleich die konventionalisierte frame: „an Hinz und Kunz weitergeben“ aufgerufen. Es werden zielkulturelle und -sprachliche frames aktiviert, die sich oft gegenseitig in einer Art „Kettenreaktion“ bedingen.

Die Aufgabe des Translators wird durch die Anwendung des Scenesand-frames Konzepts somit grob insofern erleichert, als er besonders konventionalisierte Konzepte und Schemata sichtbar machen und die entsprechenden sprachlichen Ausdrucksformen, frames in der Zielsprache, leichter abrufen kann.

Zu Recht wurde den Verfechtern des Scenes-and-frames Ansatzes jedoch vorgeworfen - und darin liegt derzeit noch seine Schwäche - dass er nicht erkläre, wie frames denn überhaupt erstellt und voneinander abgegrenzt werden können (vgl. Gerzymisch-Arbogast 1994:77). Ausgehend von dieser Kritik möchte ich im Nachfolgenden eine Frame-Typologie vorschlagen, die uns bei einer systematische(re)n Identifikation und Abgrenzung der einzelnen frames behilflich sein soll. 


\section{Theoretische Grundlagen für die Erstellung einer Frame-Typologie}

Frames gehören einer Vielzahl von Wissensbereichen an. Der Versuch, alle in Texten vorkommenden Frame-Typen auszumachen und zu systematisieren, würde den Rahmen dieses Beitrags sprengen. Auch geht es mir nicht darum, eine Taxonomie aller potentiellen Frame-Typen aufzustellen. Mir liegt vielmehr daran, aufzuzeigen, dass wir bereits über theoretisches Rüstzeug für eine erste systematische(re) Identifikation und Abgrenzung verfügen.

Das in den folgenden Abschnitten beschriebene theoretische Modell war mir bei der Analyse der Textbeispiele (Vergleich des AT mit dem ZT) als Interpretationsrahmen nützlich um mehr über die Übertragung von scenes und frames zu erfahren. Ich stütze mich also auf ein bereits existierendes Modell, das auch schon auf eine empirische Basis gegründet ist (vgl. Fallstudien von Ana Maria Rojo Lopez 2002). Das Textmaterial dient als Illustration der Theorie aber auch zur Überprüfung der Theorie, genauer gesagt, um festzustellen, ob diese Theorie für das Übersetzen wirklich relevant ist.

Den Ausgangspunkt meiner Analyse bildete Manuel de Vega's FrameTypologie (1984), die im Laufe dieser Untersuchung für unsere Zwecke abgeändert und erweitert wurde. De Vega unterscheidet zwischen fünf Frame-Typen, die auch zu den in den Kognitionswissenschaften meist untersuchten zählen: visual frames, situational frames (auch scripts genannt), domain frames, social frames sowie self-concept frames. (vgl. de Vega 1984 zit. in Rojo Lopez 2002: 316). Zur Definition:

VISUAL frames refer to the interpretation structures that take part in the configuration of objects and scenes in visual perception, e. g. the typical frame of a room includes a roof, a floor and four vertical walls SITUATIONAL frames structure, as their name indicates, information related to conventional situations, e. g. going to a restaurant, to the doctor etc. DOMAIN frames are structures that guide discourse events: introduction, exposition and denouement. SOCIAL frames describe the cognitive structures that organize our social knowledge. SELF-CONCEPT frames refer to the articulated knowledge that each person has of himself/herself. 


\subsection{Zur Erstellung einer übersetzungsrelevanten frame- Typologie}

Diese fünf frame-Typen wurden im Zuge der Analyse, wie bereits angedeutet, modifiziert und ergänzt und ergeben somit die hier vorgeschlagene übersetzungsrelevante frame-Typologie bestehend aus:

VISUAL frames, SITUATIONAL frames, TEXT TYPE frames,

SOCIAL frames, CULTURAL frames und LINGUISTIC frames.

Diese neue Klassifizierung bietet vier Unterschiede im Vergleich zu de Vega's frame-Typologie:

DOMAIN frames wurden durch TEXT TYPE frames ersetzt, da es bekannterweise wahrscheinlicher ist, Übersetzungsprobleme jeweils dort anzutreffen, wo die Struktur eines bestimmten Textes und die damit verbundenen kulturell spezifischen Textsortenkonventionen ins Spiel kommen. DOMAIN frames ist, auf die Übersetzungswissenschaft angewandt, eine zu weit gefasste und daher zu vage Kategorie.

Die Kategorie der SELF CONCEPT frames wurde nicht übernommen, da frames, die dieser Kategorie angehören würden, im untersuchten Textmaterial nicht vorgekommen sind und m. E. n. als zu spezifisch zu werten sind, als dass sie einen gesonderten Frame-Typ darstellen könnten.

Neu eingeführt wurden in der hier vorgeschlagenen frame-Typologie hingegen CULTURAL frames sowie LINGUISTIC frames - zwei Kategorien, die nach einer Einführung und gründlicheren Beschreibung der anderen vier frame-Typen, gleich im nächsten Abschnitt noch näher erläutert werden sollen.

\subsection{Begriffliche Abgrenzung und Illustration der einzelnen frame-Typen (für AS + ZS)}

\subsubsection{Visual frames}

Basierend auf der einschlägigen Literatur und die im Textkorpus vorkommenden Textbeispiele schlage ich vor, visual frames zu definieren als: Kognitive Strukturen, die der Organisation unserer visuellen Wahrnehmung dienen. Gemeint ist die visuelle Szene, wenn man sich z. B. einen im Text vorkommenden frame (sprachliche Kodierung) szenisch konkret vorzustellen versucht. 
Auf den Übersetzungsprozess übertragen, können visual frames v. a. bei der Beschreibung von Vorgängen, Personen, Szenen oder Objekten dienlich sein. Wenn wir eine Beschreibung lesen, entstehen in unserem Gehirn dank unserer visuellen Erfahrung Bilder. Visual frames funktionieren gleich wie andere frame-Typen: sie lösen Erwartungen aus und erlauben uns implizite Informationen auf der Basis der bereits vorhandenen visuellen Erfahrungen zu inferieren. Dem Translator kommt die Aufgabe zu, eine Übersetzung anzufertigen, die bei seinem Leser in der ZS eine Gesamtscene sowie Teilscenes evoziert, die denen der Beschreibung im AT möglichst nahekommen. Zur besseren Verdeutlichung des eben Gesagten hierzu einige Beispiele aus dem Textkorpus:

a) Visual frames zur Beschreibung von Vorgängen:

In einer seiner Glossen mit dem Titel: Come comperare gadgets, auf Deutsch: „Wie man sich das Leben durch Maschinchen erleichtert“, schildert Eco wieder auf besonders ironische und spöttelnde Art und Weise, welche neuen technischen, nicht immer wirklich nützlichen, Errungenschaften und Erfindungen das Leben der Menschen zu erleichtern versprechen.

E noto che gli americani, per eliminare il colesterolo, fanno jogging, e cioè corrono per ore e ore fino a che non strammazzano morti d'infarto. Pulse Trainer (dollari 59 e 95) si mette al polso, ed è colleggato tramite un filo a un cappuccetto di gomma che si infila sull'indice. Pare che quando il vostro sistema cardiocircolatorio sia sull'orlo del collasso suoni un allarme. È un progresso, se si pensa che nei paesi sottosviluppati uno si ferma solo quando ha il fiatone - che è un parametro molto primitivo, e forse per questo i bambini del Ghana non fanno jogging. E però curioso come, malgrado tale trascuratezza, essi siano quasi del tutto privi del colesterolo. Con Pulse Trainer potete correre tranquilli e, allacciandovi al petto e alla vita le due cinture di Nike Monitor, una voce elettronica, istruita da un microprocessore e da un Doppler Effect Ultra Sound, vi dice quanto avete percorso e a quale velocità (dollari 300).

Eco (1992: 93)

Man weiss, dass die Amerikaner, um ihre Fettpolster abzubauen, Jogging machen, das heisst, sie laufen Stunden, bis sie mit einem Herzinfarkt zusammenbrechen. „Pulse Trainer“ (\$59.95) wird an den Puls geschnallt und ist durch einen Draht mit einem Gummikäppchen verbunden, das man sich über den Zeigefinger stülpt. Anscheinend 
Der frame „eliminare il colesterolo“ hätte auch weniger frei und ATgetreuer übersetzt werden können, z. B die Amerikaner joggen, „um ihr Cholesterin loszuwerden“. Der Übersetzer hat sich den AS-frame jedoch szenisch konkret vorgestellt. Er hat die scene, die der ausgangssprachliche frame evoziert, visualisiert und es vorgezogen dem deutschen Leser durch einen gewählten ZS visual frame dieselbe Szene schneller und leichter zugänglich zu machen, ohne dass sich letzterer die gleiche oder eine ähnliche scene vorher erst über einen konventionellen ZS-frame (wie z. B. der nicht verbildlichten sprachlichen Ausdrucksform „um ihr Cholesterol loszuwerden“) hätte verschaffen müssen.

AS-frame $\rightarrow$ visual ZS-frame.

b) Visual frames zur Beschreibung von Personen:

In einer anderen Glosse mit dem Titel: „Come usare il tassista“, in der deutschen Version: „Wie man mit Taxifahrern umgeht“ beschreibt Eco das Problem der korrekten Interaktion mit dem Fahrer, einem Menschen, der, so Eco, den ganzen Tag lang im Stadtverkehr Auto fährt - eine Tätigkeit, die unweigerlich entweder zum Herzinfarkt oder zum Nervenzusammenbruch führen muss - und der mit seinen Fahrgästen ständig in Konflikt gerate. Im nächsten Abschnitt ist die Rede davon, wie es einem Taxikunden in Stockholm ergeht. Der Kunde wird als Fremder beschrieben, der am Strassenrand winkt (visual frame). 
A Stockholm potete chiamarli solo per telefono, perché non si fidano di uno qualsiasi che passa per strada.

Eco (1992: 115)
In Stockholm kann man sie nur telefonisch bestellen, denn dort trauen sie keinem Fremden, der am Strassenrand winkt.

Kroeber (1993: 103)

Auch in diesem Abschnitt visualisiert der Übersetzer die durch den ASframe „che passa per strada“ evozierte Szene, setzt dem ursprünglichen AS-frame ein Element aus der dabei hervorgerufenen Szene hinzu, die Geste des Herbeiwinkens eines Taxis, und bildet für den deutschen Leser einen ZS-visual frame, der letzterem ermöglicht, sich das Bild des am Strassenrand winkenden Fahrgastes gleich vor Augen zu führen. Zudem verstärkt Kroeber an dieser Stelle den Verweis Ecos auf das ununterbrochene Gespräch, das zwischen und in seinen verschiedenen Texten geführt wird und auch zur Ironie beiträgt. Durch die stärkere Verdeutlichung dieses ZS-visual frames wird der krasse Gegensatz zu den vorhergehenden ZS-frames - in denen die Rede davon ist, dass man in New York Taxis nicht einmal telefonisch bestellen könne, ausser man ist Mitglied in einem Club, man es in Paris jedoch durchaus per Telefon bestellen kann, es nur dann nicht kommen würde - besser deutlich und leichter verständlich.

$$
\text { AS-frame } \rightarrow \text { visual ZS-frame. }
$$

c) Visual frames zur Beschreibung von Objekten:

Im folgenden Essay, betitelt mit „Come reagire ai volti noti“, auf deutsch: „Wie man auf bekannte Gesichter reagiert“ wird zur Beschreibung eines Objektes wieder ein visual frame herangezogen.

Questi volti popolano la nostra memoria, abbiamo trascorso con loro molte ore davanti a uno schermo.

Eco (1992: 128)
Diese Gesichter bevölkern unser Gedächtnis, wir haben mit ihnen schon viele Stunden vor einer Kinoleinwand oder einem Fernsehbildschirm verbracht.

Kroeber (1993: 118)

An dieser Stelle hat die Tatsache, sich das Objekt „schermo“ szenisch konkret vorzustellen, geholfen, den AS-frame in der ZS zu spezifizieren. Der Übersetzer fragt sich, was denn hier unter dem AS-frame 
„schermo“ gemeint sein könne, versucht die dahinter verborgene scene hervorzurufen und für sich verständlich zu machen, um sie in einen ZSverständlichen und konkreteren visual frame umzuwandeln. Kroeber löst die Doppelkodierung auf und kommt seinem Leser entgegen, indem er präzisiert, dass es sich um eine Kinoleinwand oder einen Fernsehbildschirm handeln kann (an den Fernsehbildschirm hätte der Rezipient bei einer Übersetzung wie „Bildschirm“ wohl auch gleich gedacht, eine Kinoleinwand hätte er sich allerdings nur schwieriger und vielleicht auch erst nach längerem Nachdenken vorstellen können).

Ein weiteres aussagekräftiges Beispiel, das für die Anwendung des Scenes-and-frames Ansatzes bei der Beschreibung von Vorgängen beim Übersetzen und die systematische Einteilung der frames in die verschiedenen angegebenen frame-Typen wie z. B. in visual frames spricht, ist das Folgende - zu finden in Eco's Essay „Come usare la cuccuma maledetta“, in der deutschen Version übersetzt mit: „Wie man die vermaledeite Kaffeekanne benutzt“, in dem sich der Grossmeister der Ironie über ärgerstiftende kleckernde Kaffeekannen beschwert.

Le cuccume usate dalle persone normali - o le buone vecchie caffettiere da cui si versa direttamente la fragrante bevanda nella tazza - consentono la discesa del caffé attraverso un sottile ugello o beccuccio, mentre la parte superiore dispone di un qualsiasi dispositivo di sicurezza che la tiene chiusa.

Eco (1992: 110/111)
Die von normalen Leuten verwendeten Kannen - oder auch die guten alten Espressokannen, aus denen man sich das duftende Getränk direkt in die Tasse giesst - erlauben den Austritt der Flüssigkeit durch eine feine schnabelförmige Tülle, während der Deckel irgendeine Sicherheitsvorrichtung hat, die ihn geschlossen hält.

Kroeber (1993: 94)

Auch an dieser Stelle hat sich der Übersetzer - ausgehend vom AS frame - überlegt, was mit „la parte superiore“ gemeint sein könne und sich den Gegenstand im gegebenen Kontext szenisch konkret vorzustellen versucht. Dank seines Weltwissens hat er die im AT-frame enthaltene Information inferieren können und die durchaus zutreffende Übersetzung „Deckel“ gefunden, womit der Leser in der ZS die selbe Szene evoziert, sich mitunter sogar noch genauer vorstellen kann, wovon die Rede ist, als der Leser des Originals. 


\subsubsection{Situational frames:}

Dieser frame-Typ geht auf Schank \& Abelsons Script theory in der Künstlichen Intelligenzforschung zurück (vgl. Rojo Lopez 2002: 320ff.). Scripts sind Handlungsschemata, die Szenen beinhalten, die wiederum aus einer Reihe von Ereignissen bestehen (vgl. auch Schwarze, 1992: $89 \mathrm{ff}$.). Situational frames, wie ihr Name schon sagt, stellen somit Informationsverarbeitungsprozesse dar, die an Situationen oder Handlungen gebunden sind, genauer gesagt, an Standardsituationen oder -handlungen.

Die Szene BESTELLEN als Teil des Schemas RESTAURANTBESUCH kann die Ereignisse X (KELLNER HERBEIWINKEN), Y (AUFTRAG ERTEILEN) usw. involvieren. Diese Ereignisse inkludieren gleichzeitig Rollen und Requisiten wie KELLNER, GAST, TISCH und SPEISEKARTE. Die nicht explizit erwähnten Handlungen (Essen usw.) werden mental durch die Aktivierung des Handlungsschemas bereitgestellt.

Situational frames können ausserdem aus verschiedenen Perspektiven beschrieben werden: So wird die aus der Sicht des Kellners beschriebene Handlungskette nicht dieselbe sein, wie die aus der Sicht des Gastes beschriebene. Im Zusammenhang mit der Übersetzung bedeutet dieser letzte Punkt, dass gerade ein Perspektivenwechsel - wie auch im Verlauf unserer Versuchsreihen anhand von Protokollen des Lauten Denkens und der Verwendung der Software Translog (Heiden 2004) festzustellen war - bei schwierig zu übersetzenden Stellen oft sehr kreative Lösungen hervorbringen kann (vgl. auch Kussmaul, 2002).

Ist kein Schema explizit erwähnt, wählt der Übersetzer aufgrund seines Vorwissens ein passendes aus: So wird z. B. das folgende situational frame (hier: die Standardsituation) „Ich suchte wie wild nach der Fahrkarte, als der Schaffner ins Abteil trat“ einem BAHNFAHRT-Schema (Standardhandlung) zugerechnet werden.

Graesser et al. (1979) und Bower (1979) konnten durch experimentelle Studien nachweisen, dass scripts (was somit auch für die hier als situational frames bezeichneten sprachlichen Rahmen Gültigkeit hat) kulturspezifisch sind. Sie haben im Laufe ihrer Untersuchungen u.a. entdeckt, dass das script ARZTBESUCH in Amerika im Vergleich zu Spanien unterschiedlich abläuft: In Amerika legen die Patienten ihre Kleidung ab, schildern ihre Krankengeschichte einer Krankenschwester und 
sehen meist erst dann einen Arzt, wohingegen die Patienten in Spanien sich gewöhnlich gleich an den Arzt wenden, um ihm ihr Problem zu schildern. Nachdem situational frames kulturell unterschiedlich ablaufen, muss dies der Translator zunächst erkennen (dank seiner Erfahrung im Zusammenhang mit bestimmten Standardsituationen und -handlungen gelingt ihm dies in den meisten Fällen auch leicht) und sieht sich anschliessend vor der Herausforderung, kreative Strategien zur Kompensation zu finden. (vgl. Kussmaul 2002: Kreative Typen des Übersetzens).

Zur besseren Veranschaulichung von situational frames auch hierzu wieder ein paar Beispiele aus unserem Textkorpus:

In der bereits für ein anderes Beispiel herangezogenen Glosse „Come usare il tassista“, auf deutsch: „Wie man mit Taxifahrern umgeht.“ schreibt Eco ganz am Schluss:

Ovunque, per riconoscere un tassista, c’è un mezzo infaillibile. E quella persona che non ha mai il resto.

Überall gibt es ein unfehlbares Mittel, einen Taxifahrer zu erkennen: Er ist Eco (1992: 115) immer derjenige, der nie herausgeben kann.

Kroeber (1993: 104)

Dank dem script TAXIFAHREN bestehend aus den Szenenelementen TAXI HERBEIWINKEN bzw. TAXI BESTELLEN (je nach Land), INS TAXI EINSTEIGEN, FAHREN, ANHALTEN, ZAHLEN, ist wenn auch nicht explizit genannt - aus dem Weltwissen des Übersetzers erschliessbar, dass es sich bei dem AS-situational frame „il resto“ um den ZS-frame „Wechselgeld“ handeln muss.

Ein anderes ansprechendes Beispiel für einen situational frame können wir in Eco’s Essay „Come mangiare in aereo“, in der deutschen Version übersetzt mit „Wie man in Flugzeugen speist“ finden, in dem sich Eco lästernd darüber äussert, dass in Flugzeugen auf Langstreckflügen kurioserweise jedes Mal Speisen serviert werden, die sich nur schwer essen lassen, so z. B. durchbratenes Fleisch in grosszügig servierter brauner Sauce, enorme Portionen gekochter Tomaten, feingeschnittenes und in Wein getränktes Gemüse, Reis und ungreifbare Erbsen in viel eigenem Saft. 
Si aggiunga che i piselli, in aereo, sono regolarmente serviti solo quando l'aeremobile entra in turbolenza e il capitano raccomanda di allanciare le cinture.

Eco (1992: 101)
Hinzu kommt, dass die Erbsen im Flugzeug mit schöner Regelmässigkeit immer nur dann serviert werden, wenn das Flugzeug gerade in eine Turbulenz gerät und der Kapitän empfiehlt, die Sicherheitsgurte anzulegen.

Kroeber (1993: 76)

Die wortwörtliche Entsprechung des AS-frames „cintura“ fände sich im deutschen ZS-frame „Gürtel“, „Sicherheitsgurt“ hiesse auf Italienisch: „la cintura di sicurezza“. Dank dem script REISEN IM FLUGZEUG wird jedoch verständlich, dass es sich bei dem Gürtel um einen eigens dafür vorgesehenen Sicherheitsgurt handeln muss. Diese nicht explizit erwähnte Information wird mental durch die Aktivierung des bekannten Handlungsschemas bereitgestellt.

Ein letztes Beispiel stammt aus Eco’s Glosse: „Come scrivere un’introduzione“, auf deutsch: „Wie man ein Vorwort schreibt.“ Ziel dieses Streichholzbriefchens ist es wieder, auf besonders sarkastische Art zu erklären, wie man ein Vorwort gestaltet, warum man ein Vorwort schreiben muss, was es enthalten soll und wie v. a die Danksagungen zu gestalten sind. Nur nebenbei bemerkt, meint Eco in dem Zusammenhang, übrigens auch recht spöttelnd: „Die Gewandtheit der Danksagungen charakterisiert den Wissenschaftler von Rang.“

Un particolare ringraziamento al direttore amministrativo Milvia Fiorani che con la sua continua e mensile assistenza ha provveduto alla continuazione della mia ricerca.

Eco (1992: 106)
Besonderer Dank sei der Verwaltungsdirektorin Milvia Fiorani gesagt, die mit ihren fortgesetzten monatlichen Überweisungen für die Fortsetzung meiner Studien gesorgt hat.

Kroeber (1993: 85)

Die Szene EINEN BEITRAG SCHREIBEN als Teil des Handlungsschemas FORSCHUNG BETREIBEN involviert - da es sich in diesem konkreten Fall um eine lukrative Tätigkeit handelt (Eco schreibt für das wöchentliche römische Nachrichtenmagazin L’Espresso) - das Ereignis, dafür auch bezahlt zu werden. Diese Information ist im Text nicht explizit enthalten, dem Übersetzer durch die Aktivierung des bekannten Handlungsschemas jedoch abrufbar. Dies erlaubt ihm, die hinter 
dem AS-situational frame „assistenza“ steckende scene in diesem spezifischen situationsgebundenen Kontext aufzurufen und sie durch eine angemessene Übertragung (Konkretisierung des AT-frames) auch dem Leser der ZS zugänglich zu machen.

\subsubsection{Social frames}

Social frames können anlehnend an Hamilton (1981), Schank \& Abelson (1977) wie folgt definiert werden: Kognitive Strukturen, die der Organisation unseres sozialen Wissens dienen. De Vega (1986) unterscheidet die folgenden social frame-Typen: generic frames, themes, role themes, interpersonal themes, life themes, ideologies and systems of beliefs, die mir für meine Untersuchung jedoch als zu vage und generell gefasst schienen. Eine translationsrelevante Unterteilung von social frames findet sich bei Rojo Lopez (2002: 327). Sie schlägt vor, social frames in vier frame-Typen zu unterteilen, die, wie sich auch im Zuge meiner Analyse herausgestellt hat, zu Übersetzungsproblemen führen können. Es handelt sich um AS-frames in Verbindung mit der geographischen Herkunft (geographic origin frames, vgl. Rojo Lopez 2002; z. B. bei der Übersetzung von Dialekten ), mit dem sozialen Status (social status frames, vgl. Rojo Lopez 2002, welche die Aktivierung von Kulturwissen des Übersetzers erfordern, im Zusammenhang mit der sozio-ökonomischen Herkunft eines Sprechers z. B. bei der Übertragung von verschiedenen Sprachregistern) sowie mit interpersonalen Relationen (interpersonal frames, vgl. Rojo Lopez 2002, welche das Wissen des Translators im Zusammenhang mit sozialen und affektiven Beziehungen der Mitglieder einer Sprachgemeinschaft organisieren z. B. bei der Übersetzung verschiedener Formen der Anrede, sowie umgangssprachlicher Ausdrücke und Kollokationen, dem Umgang mit Tabuwörtern usw.).

\subsubsection{Cultural frames}

Neu eingeführt werden sollen in der hier vorgeschlagenen frame-Typologie cultural frames, da kulturspezifische Elemente in Texten äusserst häufig vorkommen und daher m. A. n. auch eine eigene frame-Kategorie verdienen. Zudem können cultural frames nicht immer den anderen von De Vega vorgeschlagenen frame-Typen zugeordnet werden. Defi- 
niert werden sollen cultural frames als: Kognitive Strukturen die unser Kulturwissen organisieren.

Für den Übersetzer bedeutet ein im AT vorkommender cultural frame, dass hinterfragt werden muss, welche scene hinter dem frame steckt und ob dieser cultural frame eins zu eins übertragen beim Leser in der ZS dieselbe scene evozieren wird, wie er dies in der AS tut, oder nicht. Für den Fall, dass der ZS Leser aufgrund seiner Zugehörigkeit zu einer anderen Kultur mit dem ZS frame eine ganz andere Vorstellung assoziiert als der Leser in der Ausgangskultur oder als vom Autor beabsichtigt, muss der Übersetzer diesen cultural frame dekodieren und in der ZS neu enkodieren bis er die passenste scene hinter dem neuen ZS frame gefunden hat. Scenes und frames laufen - wie wir weiter oben bereits ausgeführt haben - eben kulturell unterschiedlich ab. Der Übersetzer sieht sich daher jeweils vor die Aufgabe gestellt, AS-frames an die Kulturspezifika des jeweiligen Landes anzupassen. Beispiele für cultural frames sind natürlich zahlreiche zu finden.

Bei dieser Gelegenheit möchten wir die unter Punkt 2.3.2. bereits vorgestellten Beispiele in Erinnerung rufen. Dabei ging es darum dem Leser zusätzlich zu den theoretischen Erläuterungen zum Scenes-andframes Ansatz vor allem auch Beispiele zu präsentieren. Sie alle stellten Beispiele für cultural frames dar, bei denen es jeweils erforderlich war, die AS-frames an die kulturellen Unterschiede in der ZS anzupassen.

1. Passage aus: „Come fare vacanze intelligenti“ (1992: 75) / „Wie man intelligente Ferien macht“ (1993: 31)

tre dei sei volumi einaudiani del Ramusio

$\Downarrow$

drei Bände der sechsbändigen Einaudi-Ausgabe der „Navigazioni et viaggi“ des humanistischen Geographen Gian Battista Ramusio

2. Passage aus: „Come fare vacanze intelligenti“ (1992: 75) / „Wie man intelligente Ferien macht“ (1993: 30)

l'originale tedesco delle Affinità elettive

$\Downarrow$

das englische Original des „Tristram Shandy 
Die von Rojo Lopez 2002 als institutional frames bezeichneten sprachlichen Formulierungen, die letztere nicht unbedingt einleuchtend in die Kategorie der social frames einordnet, sollen in der hier vorgeschlagenen Typologie eine mögliche Untergruppe von cultural frames darstellen. Unter institutional frames ist die (eben auch wieder jeweils kulturspezifische) Benennung von Einrichtungen zu verstehen, die das Wissen des Translators in Bezug auf länder- und kulturspezifische Einrichtungen, Systeme oder Objekte abruft.

Ein Beispiel für einen AS-institutional frame findet sich in der Glosse „Come presentare un catalogo d'arte“, auf deutsch: „Wie man einen Ausstellungskatalog bevorwortet“:

Se si tratta di una mostra si paesaggi alpini stile Segantini, non è necessario, anzi è dannoso, scrivere sur New Yorker ed è più opportuno essere preside del locale istituto magistrale.

Eco (1992: 67)
Handelt es sich zum Beispiel um eine Ausstellung von alpinen Landschaften im Stil Segantinis, so ist es nicht nötig, ja sogar schädlich, im New Yorker zu schreiben und es empfiehlt sich eher, Leiter der örtlichen Volkshochschule zu sein.

Kroeber (1993: 15)

\subsubsection{Linguistic frames}

Zuletzt möchte ich noch vorschlagen, ergänzend zu visual frames, texttype frames, situational frames, social frames sowie cultural frames einen gesonderten frame-Typ einzuführen - den der linguistic frames, da es beim Übersetzen nicht nur gilt AS-frames an die spezifische Art der Wahrnehmung, an Situations- bzw. Handlungsschemata, soziale und kulturelle Eigenheiten sowie an Textsorten, -typen und -konventionen anzupassen, sondern Adaptierungen manchmal auch vorgenommen werden müssen, um das Sprachsystem des jeweiligen Ziellandes zu respektieren. Linguistic frames sind in diesem Sinne zu definieren als: Kognitive Strukturen, welche der Organisation unseres Sprachwissens dienen. Sehen wir uns zur besseren Verständlichkeit auch hier wieder ein Textbeispiel an, das dieses Mal aus Eco’s Glosse: „Come si organizza una biblioteca pubblica“" stammt, in der deutschen Ausgabe übersetzt mit „Wie man eine öffentliche Bibliothek organisiert“: 
Possibilmente l'ortografia, nei due cataloghi (acquisizioni recenti e antiche) deve essere diversa; per esempio nelle acquisizioni recenti retorica va con un $\mathbf{t}$, in quella antica con due t; Cajkovskij nelle acquisizioni recenti col $\mathrm{C}$, mentre nelle acquisizioni antiche alla francese, col Tch.

Eco (1992: 73)
Nach Möglichkeit sollte die Orthographie in den beiden Bücherkatalogen (Neuerwerbungen und alter Bestand) verschieden sein: beispielsweise Begriffe wie „Code“, in dem einen mit $C$, in dem anderen mit K, oder Namen wie Tschaikowski bei Neuerwerbungen mit einem $C$, bei den anderen mal mit $\mathrm{Ch}$, mal mit Tch.

Kroeber (1993: 26)

Bei beiden von Eco gewählten Beispielen musste Burkhard Kroeber adaptieren. Im ersten Fall hatte er gar keine andere Wahl als einen ganz anderen, nicht bedeutungsgleichen linguistic frame heranzuziehen, da sich „Rhetorik“ im Deutschen nicht so schreibt wie im Italienischen und auch etymologisch keine mit dem Italienischen AT-frame zu vergleichende Entwicklung durchgemacht hat. Im zweiten Fall war er gezwungen, das Beispiel an die deutschen Orthografieregeln anzupassen und nach anderen Schreibweisen des italienischen AS-frame „Cajkovskij“ im Verlauf der deutschen Sprachentwicklungsgeschichte zu suchen, um den durch die AS-scene provozierten Witz beizubehalten und dem deutschsprachigen Leser eine ähnliche scene aufzubauen.

\section{Der Scenes-and-frames Ansatz: Ausgangspunkt für eine kognitive übersetzungsprozedurale Konzeption?}

Auf der Grundlage der theoretischen Ausführungen in den letzten Kapiteln und inspiriert durch Giorgio Floro's Modell (2001) zur Übersetzung von kulturellen Konstellationen möchte ich abschliessend noch aufzeigen, inwiefern uns der scenes-and-frames Ansatz und die vorhin vorgestellte Frame-Typologie - ausgeweitet auf ein übersetzungsprozedurales Verfahren - als konkrete methodologische Hilfestellung beim Übersetzen dienlich sein kann.

Das von mir zu diesem Zweck vorgeschlagene Verfahren soll in drei Phasen ablaufen: einer Rezeptions-, einer Transfer- und einer Reproduktionsphase.

Die Rezeptionsphase dient der Identifizierung von scenes und frames im Ausgangstext und wird in vier Schritten vollzogen. Der erste Schritt besteht in der erstmaligen Lektüre des Ausgangstextes. Dabei werden die für den Text relevanten scences und frames ermittelt, die der Text 
entweder explizit oder implizit bereitstellt. In Schritt 2 folgt dann die systematische Auflistung und Erstellung dieser konzeptuellen Systeme, bestehend aus Konzepten und Schemata. In Schritt 3 wird der Text ein zweites Mal durchgelesen und die einzelnen Systeme nacheinander betrachtet. Dabei können die Textstellen, die bei der Übertragung von scenes und frames zu Schwierigkeiten führen können, markiert werden. Die Identifizierung und Einordnung von scenes und frames in die von uns vorgeschlagene frame-Typologie geschieht im Schritt 4 .

Die zweite Phase wird Transferphase genannt. In dieser Phase werden zunächst (Schritt5) anhand der vorgeschlagenen frame-Typologie die ZS konzeptuellen Systeme bestehend wieder aus scenes und frames erstellt. Der Schritt 6 besteht darin ausgangssprachliche und zielsprachliche scenes und frames auf Systemebene miteinander zu vergleichen. Dabei werden volle Übereinstimmung, teilweise Übereinstimmung und NichtÜbereinstimmung ermittelt, sowie die Tatsache, ob diese intendiert oder nicht-intendiert ist. Im Schritt 7 folgt eine Kompatibilitätsprüfung. Dabei soll herausgefunden werden, inwiefern die gefundenen frames und die dahinterstehenden scenes mit dem System der Zielkultur auch kompatibel sind, dies freilich wieder unter Berücksichtigung des Vorwissens und der Erwartungen des Zielpublikums. Für den Fall, dass manche Textstellen mit dem System der Zielkultur inkompatibel sind, ist mit Verständnisschwierigkeiten zu rechnen. Das Ergebnis der Transferphase ist die systematische Ausmachung von Unterschieden und Kompatibilitäten der ausgangssprachlichen und zielsprachlichen konzeptuellen Systeme.

In einer letzten Phase, der Reproduktionsphase, wird eine Hierarchisierung der gefundenen ZS frames vorgenommen und und die angemessenste Lösung unter Berücksichtigung des Hintergrundwissens und der Erwartungen des Zielpublikums ausgewählt. In einem letzten Schritt findet dann die Neuvertextung statt.

Die im Rahmen dieses Beitrags vorgeschlagene Frame-Typologie erhebt freilich keinen Anspruch auf Vollständigkeit und soll im Verlauf weiterer Untersuchungen anhand umfangreicherer Textkorpora weiter ergänzt und notwendigenfalls auch abgeändert werden. Die Typologie sowie das hier vorgestellte mögliche übersetzungsprozedurale Verfahren aus kognitiver Sicht sollen weiters primär als Diskussionsgrundlage dienen und - so wäre dies zumindest wünschenswert - auch in der Praxis 
und in der Übersetzungsdidaktik (als methodogische Hilfestellung für Studenten der Translationswissenschaften) angewandt und überprüft werden.

\section{Bibliographie}

\section{Primärquellen}

Eco, Umberto 1998: Il secondo diario minimo. I Grandi Tascabili, Milano RCS Libri S.p. A. (prima edizione 1992 Bompiani: Milano).

Eco, Umberto 2002: Wie man mit einem Lachs verreist und andere nützliche Ratschläge. 9. Auflage (1. deutschsprachige Auflage 1993: Carl Hanser Verlag: München, Wien). Aus dem Italienischen von Burkhard Kroeber und Günther Mammert. München: DTV.

Eco, Umberto 2002: Gesammelte Streichholzbriefe. Ungekürzte Ausgabe. (1. deutschsprachige Auflage 1990/95: Carl Hanser Verlag: München, Wien). Aus dem Italienischen von Burkhard Kroeber. München: DTV.

\section{Sekundärquellen}

Albrecht, Jörn 1998: Literarische Übersetzung. Geschichte - Theorie - Kulturelle Wirkung. Wissenschaftl. Buchgesellsch.

Brodbeck, Karl-Heinz 1999: Entscheidung zur Kreativität. Primus Verlag, Wissenschaftliche Buchgesellschaft: Darmstadt.

Feyrer, Cornelia 2001: „Interaktionsmanagement und Translation: von scenes und frames und der Begegnung mit dem Teufel“. In TcT 15, NF 5, 2001, 1-76.

Floros, Giorgios 2001: Kulturelle Konstellationen in Texten. Jahrbücher Übersetzen und Dolmetschen. Band 3/2003. Tübingen: Narr

Fillmore, Charles J. 1977: „Scenes-and-frames semantics“. In Zampolli, Antonio (ed.) Linguistic structure processing. Amsterdam: North Holland, 55-81

Graesser, A. C. et al. 1979: „Recognition memory for typical and atypical actions in scripted activities: Tests of a script pointor + tag hypothesis“. In Journal of Verbal Learning and Verbal Behavior; zit. in P. Kussmaul (2000).

Guilford, Joy Peter 1975: „Creativity: A Quarter Century of Progress“. In Taylor, I.A. I Geztels, J.W. (Hrsg.): Perspectives in Creativity. Chicago: Aldine, 37-59.

Heiden, Tanja 2004: „Wenn Translate aus dem Rahmen fallen oder Kreatives Problemlösen beim Übersetzen. Eine empirische Untersuchung des Übersetzungsprozesses ausgehend vom Scenes-and-frames Ansatz unter Verwendung von Translog 2000“. Mémoire zur Erlangung eines DEA (Diplôme d'études approfondies) en traduction an der Ecole de Traduction et d'Interprétation der Universität Genf. 
Hoenig, Hans; Kussmaul, Paul 1982: Strategie der Übersetzung. Ein Lehr- und Arbeitsbuch. Tübingen: Narr.

Hoenig, Hans 1986: „Übersetzen zwischen Reflex und Reflexion. Ein Modell der übersetzungsrelevanten Textanalyse“. In Snell-Hornby, Mary (Hrsg.), Übersetzungswissenschaft. Eine Neuorientierung. Tübingen: Francke UTB, 230-251.

Kussmaul, Paul 1995: Training the Translator. Amsterdam / Philadelphia: Benjamins

Kussmaul, Paul 1997: „Die Rolle der Psycholinguistik und der Kreativitätsforschung bei der Untersuchung des Übersetzungsprozesses“. In Fleischmann, Eberhard / Kutz, Wladimir / Schmitt, Peter A. (Hrsg.): Translationsdidaktik. Grundfragen der Übersetzungswissenschaft. Tübingen: Narr, 605-611.

Kussmaul, Paul 1999: „Die Übersetzung als kreatives Produkt“. In Gil, Alberto/ Haller, Johann / Steiner, Erich / Gerzymisch-Arbogast, Heidrun (Hrsg.): Modelle der Translation. Grundlagen für Methodik, Bewertung, Computermodellierung. Frankfurt am Main: Peter Lang, 177-189.

Kussmaul, Paul 2000: „Types of creative translating“. In Chesterman, Andrew / Gallardo, Navidad / Gambier, Yves (Hrsg.): Translation in Context. Selected Contributions from the EST Congress, Granada 1998, Amsterdam / Philadelphia: Benjamins, 117-126.

Kussmaul, Paul 2000: Kreatives Übersetzen. Studien zur Translation. Tübingen: Stauffenburg.

Reiss, Katharina und Vermeer, Hans 1991: Gundlegung einer allgemeinen Translationstheorie. Tübingen: Niemeyer (Linguistische Arbeiten 147).

Reiss, Katharina 1995: „Adäquatheit und Äquivalenz als Schlüsselbegriffe der Übersetzungstheorie und -praxis“. In Snell, Hornby, Mary / Kadric, Mira (Hrsg.): Grundfragen der Übersetzungswissenschaft. Wiener Vorlesungen von Katharina Reiss. Wien: WUV-Universitätsverlag, 107-129.

Riccardi, Alessandra 1998: „Interpreting Strategies and Creativity“. In Beylard-Ozeroff, Ann / Kralowa, Jana / Moser-Mercer, Barbara (Hrsg.) (1998): Translators Strategies and Creativity. Amsterdam / Philadelphia: John Benjamins.

Rojo Lopez, Ana Maria 2002: „Applying Frame Semantics to translation: a pratical example“. In Meta, XLVII, 3, 2002.

Rojo Lopez, Ana Maria 2002: „Frame Semanics and the Translation of Humour“. In Babel 48: 1, 34-77.

Rosch, Eleanor 1973: „On the Internal Structure of Perceptual and Semantic Categories“. In Moore, Timothy E. (ed.): Cognitive Development and the Acquisition of Language. New York: 111-144.

Rouquette, Michel-Louis 1997: La créativité. Paris: Presses universitaires de France, Que sais-je.

Schank, Roger C., and Robert P. Abelson 1977: Scripts, plans, goals, and understanding: An inquiry into human knowledge structures. Hillsdale, NJ: Lawrence Erlbaum; zit. in P. Kussmaul (2000). 
Schwarz, Monika 1996: Einführung in die Kognitive Linguistik. 2. Auflage, UTB für Wissenschaft, Francke Verlag Tübingen und Basel.

Schwalger, Petra 1997: „Die Kriminalisierung des Ciderverkaufs - Strategien des Kulturtransfers anhand der scenes-and-frames Theorie“. In Grbic, Nadja / Wolf, Michaela (Hrsg.) Text - Kultur - Kommunikation: Translation als Forschungsaufgabe: Festschrift aus Anlass des 50jährigen Bestehens des Instituts für Übersetzerund Dolmetscherausbildung an der Universität Graz / - Tübingen: Stauffenburg Verlag.

Snell-Hornby, Mary 1988: Translation Studies: An Integrated Approach. John Benjamins Publishing.

Snell-Hornby, Mary 2001: „Scenes, frames, Skopos: Sinn und Leistung des ganzheitlichen Ansatzes in der Translation“. In TcT 15, NR 5, 2001, 2, 151-163.

Stolze, Radegundis: Übersetzungstheorien. Eine Einführung. Tübingen: Narr 1994 (1982 erstm. Ersch.).

Vannerem, Mia und Snell-Hornby, Mary 1994: „Die Szene hinter dem Text: scenes-andframes semantics in der Übersetzung“. In Snell-Hornby, Mary (ed.) Übersetzungswissenschaft. Eine Neuorientierung. Tübingen - Basel: Francke (UTB 1415) 184205.

Vega de, Manuel 1984: Introduccion a la Psicologia Cognitiva. Madrid: Alianza

Vermeer, Hans J (1978): „Ein Rahmen für eine allgemeine Translationstheorie“. In Lebende Sprachen 23.3, 99-102.

Vermeer, Hans J. und Witte, Heidrun 1990: Mögen Sie Zistrosen? Scenes und frames und channels im translatorischen Handeln. Groos: Heidelberg (TEXTconTEXT Beispielheft 3).

Wilss, Wolfram 1988: Kognition und Übersetzen. Zur Theorie und Praxis der menschlichen und der maschinellen Übersetzung. Tübingen: Niemeyer. 


\title{
When Talk is a Science...
}

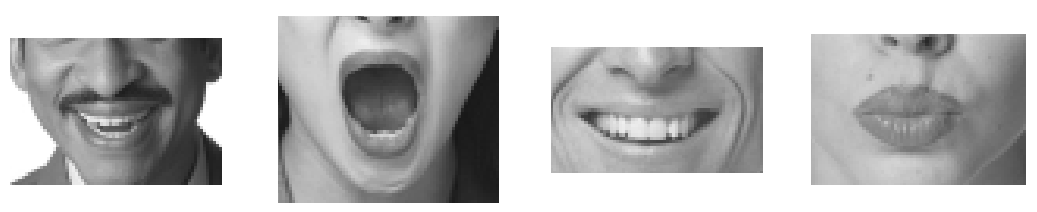

\section{Linguistics and Language Behavior Abstracts}

Comprehensive, cost-effective, timely coverage of current ideas in linguistics and language research

Abstracts of articles, books, and conference papers from nearly 1,500 journals published in 35 countries; citations of relevant dissertations as well as books and other media.

Available in print or electronically through the Internet Database Service from Cambridge Scientific Abstracts (www.csa.com).

Contactsales@csa.com for trial Internet access or a sample issue.

\section{Linguistics \& Language Behavior Abstracts}

\author{
Published by CSA
}

